\title{
Studies of dense cores in regions of massive star formation
}

\section{Structure and kinematics of dense cores from ammonia observations ${ }^{\star}$}

\author{
I. Zinchenko ${ }^{1,2}$, Th. Henning ${ }^{3}$, and K. Schreyer ${ }^{3}$ \\ 1 Institute of Applied Physics of the Russian Academy of Sciences, 46 Uljanov str., 603600 Nizhny Novgorod, Russia \\ 2 Helsinki University Observatory, Tähtitorninmäki, P.O. Box 14, FIN-00014 University of Helsinki, Finland \\ 3 Max Planck Society, Research Unit "Dust in star-forming regions", Schillergäßchen 3, D-07745 Jena, Germany
}

Received July 29; accepted November 26, 1996

\begin{abstract}
We present results of the observations of 17 molecular clouds associated with bright FIR sources in the $\mathrm{NH}_{3}(1,1)$ and $(2,2)$ lines with the 100 -m radio telescope in Effelsberg. The lines were detected in 11 clouds and 10 of them have been mapped in these lines. The kinetic temperatures, sizes, masses and mean densities of the ammonia cores have been derived.

For most of the detected clouds the masses derived under the assumption of a relative ammonia abundance of $\chi\left(\mathrm{NH}_{3}\right)=310^{-8}$ are close to virial masses. However, in $\mathrm{S} 88 \mathrm{~B}$ the ratio $M / M_{\mathrm{vir}}$ is only $\sim 0.1$ and in a few other cases this ratio is significantly lower than unity which may indicate ammonia underabundance. Almost all objects with the signs of underabundance are among the most luminous IR sources in our sample.

Most of the mapped cores are elongated with noticeable velocity gradients along the major axis. The most prominent example is S 255 . S 87 has a two-component structure with 2 distinct velocity components which overlap partly spatially. In about half of the mapped sources the $\mathrm{NH}_{3}$ line widths increase near the peaks of the ammonia emission.

The kinetic temperatures are centrally peaked. They reach $20-28 \mathrm{~K}$ in the centre and drop to $15-20 \mathrm{~K}$ at the edges of the ammonia emitting regions.

In $\mathrm{S} 76 \mathrm{E}$ a weak extended emission in the $(1,1)$ line at the velocity blue-shifted by $\sim 5 \mathrm{~km} \mathrm{~s}^{-1}$ relative to the core emission was detected. Hyperfine intensity anomalies in the $(1,1)$ transition were found in $\mathrm{S} 199$ in addition to the known case of these anomalies in S 87.
\end{abstract}

Send offprint requests to: I. Zinchenko

* Tables 5-14 are only available in electronic form at the CDS via anonymous ftp to cdsarc.u-strasbg.fr (130.79.128.5) or via http://cdsweb.u-strasbg.fr/Abstract.html
Key words: stars: formation - ISM: clouds - ISM: molecules — radio lines: interstellar

\section{Introduction}

Systematic studies of dense molecular cores in regions of high mass star formation (HMSF) are of great importance for understanding their general properties. In comparison with low mass star-forming regions, extended samples of which have been observed in various molecular lines, only a few, rather arbitrarily selected cores associated with HMSF regions have been investigated in some detail (for a review see, e.g., Walmsley \& Güsten 1994).

The goal of the present study was twofold: (1) to investigate in more detail the structure of selected HMSF cores and (2) to extend our previous ammonia survey of bright FIR sources (Schreyer et al. 1996; hereafter Paper I) to another part of the Galaxy.

We selected $(1,1)$ and $(2,2)$ ammonia lines for this study because they allow for the derivation of the core temperatures and can be directly compared with our previous results. At the same time we bear in mind that the ammonia distribution in many cases differs significantly from distributions of other high-density tracers (e.g. Zhou et al. 1989, 1991; Zinchenko et al. 1994, hereafter Paper II). The reasons for this difference should be further investigated.

This work is a continuation of the investigation of dense cores in molecular clouds associated with Sharpless $\mathrm{HII}$ regions in the $J=1-0 \mathrm{HCN}$ and $\mathrm{HCO}^{+}$lines by Burov et al. (1988), Zinchenko et al. (1989) and Zinchenko et al. (1990). Several clouds from this sample have been subject to a detailed study in the CS $J=2-1$ line in Paper II. A part of them has been mapped in the $(1,1)$ and $(2,2) \mathrm{NH}_{3}$ lines by Harju et al. (1993). Ammonia is a convenient "thermometer" for dense cores and provides 
information on their density and kinematics too. In addition, the comparison of the CS and ammonia maps can be informative for understanding the cloud structure and chemistry. Thus, similar ammonia data for the remaining cores were highly desirable.

This sample overlaps partly with the sample of bright FIR sources in the outer Galaxy which has been investigated already in various molecular lines and continuum emission by Snell et al. (1988, 1990), Carpenter et al. (1990, 1993) and Henning et al. (1992). Recently we surveyed these objects in the $(1,1)$ and $(2,2) \mathrm{NH}_{3}$ lines and in the $J=1-0 \mathrm{HCO}^{+}$line (Paper I). The detection rate in the $\mathrm{NH}_{3}(1,1)$ line was $37 \%$. An extension of this survey to the inner Galaxy should be important taking into account that there are hints on a galacto-centric dependence of the mean core density (e.g. Zinchenko 1995). We selected targets for the present study from the list of FIR sources in Sharpless clouds using the same criterion as in Paper I, i.e. the flux in the IRAS Point Source Catalogue at $100 \mu \mathrm{m} F(100 \mu \mathrm{m})$ should be larger than 500 Jy applying it to another range of right ascension. Of course, there is a number of other powerful FIR sources in the inner Galaxy which are not associated with Sharpless clouds.

Most of the cores surveyed in ammonia lines in Paper I have not been mapped in these lines. However, in order to obtain a complete physical representation of these objects such maps are very important. Thus, we included in the list several strong sources from this survey.

Here we present the results of the observations of 17 clouds in the $(1,1)$ and $(2,2) \mathrm{NH}_{3}$ lines. Ten of them (all sufficiently strong detections) have been mapped in these lines. The relevant physical properties are estimated and briefly discussed. A more detailed analysis of the data on some individual objects will be a subject of subsequent publications.

In Sect. 2 we describe the observations and data analysis. In Sect. 3 we present the observational results and derive the physical parameters of the sources. In Sect. 4 the results are discussed and in Sect. 5 we present our conclusions.

\section{Observations and data analysis}

\subsection{Observational procedure}

The observations were performed in April 1995 with the Effelsberg 100-m radio telescope. The $\mathrm{NH}_{3}(1,1)$ and $(2,2)$ lines (frequencies equal $23694.495 \mathrm{MHz}$ and $23722.633 \mathrm{MHz}$, respectively) were observed simultaneously using a $K$-band maser amplifier. The system temperature in the main beam temperature scale (see below) was $\sim 150 \mathrm{~K}$. The spectrometer was a 1024 channel autocorrelator split into 2 halves of $12.5 \mathrm{MHz}$ each. The velocity resolution was $0.31 \mathrm{~km} \mathrm{~s}^{-1}$.

The antenna HPBW was $\sim 40^{\prime \prime}$. The observations were performed in the position-switching mode with the refer- ence position displaced by $\sim 40^{\prime}$ to the west. The integration times were $2-5$ min. Initially, $3 \times 3$ point maps with $40^{\prime \prime}$ spacing were obtained for most sources. Then, the mapping was continued with the same spacing according to the initial results and extended normally to $\lesssim 10 \%$ of the peak antenna temperature value. Pointing was checked periodically by observations of nearby continuum sources; the pointing accuracy was $\sim 5^{\prime \prime}$.

We express the results in units of main beam brightness temperature $\left(T_{\mathrm{MB}}\right)$. The main beam efficiency of the antenna at this frequency is $\sim 0.3$. The primary calibration source was NGC 7027 for which we assumed the flux density as given by Ott et al. (1994). The elevation gain correction for point sources (Altenhoff, private communication) was applied to the data. We saw no variations of the beam width with the elevation, so this correction should be applicable to extended sources too. The correction factor was $\lesssim 1.1$ in the elevation range of our observations.

\subsection{Source list}

The objects for the observations (Table 1) were selected primarily from the list of dense molecular clouds associated with Sharpless H II regions studied earlier in the $J=1-0 \mathrm{HCN}, \mathrm{H}^{13} \mathrm{CN}, \mathrm{HCO}^{+}$and $\mathrm{H}^{13} \mathrm{CO}^{+}$lines by Burov et al. (1988), Zinchenko et al. (1990) and Pirogov et al. (1995). The selection was made according to the criteria similar to those used in Paper I, i.e. the flux in the IRAS Point Source Catalogue at $100 \mu \mathrm{m} \mathrm{F}(100 \mu \mathrm{m})$ should be larger than $500 \mathrm{Jy}$. However, the right ascension of $12^{\mathrm{h}}-24^{\mathrm{h}}$ instead of $0-12^{\mathrm{h}}$ as in Paper I was considered. In addition, several clouds from Papers I and II were included for detailed mapping in the ammonia lines. Most of these objects are associated with $\mathrm{H}_{2} \mathrm{O}$ masers.

Almost all of the selected targets represent sites of high mass star formation except S 145 (L 1206) and RNO 1B (L 1287) where mostly intermediate mass stars are born.

The source coordinates are presented in Table 1 together with the estimates of the galacto-centric distance of the source $\left(R_{\mathrm{G}}\right)$, its distance from the earth $(d)$ and its height above the galactic plane $(z)$. We adopted IRAS positions as central coordinates of the sources. For some clouds very different distance estimates have been published. For the sake of the homogeneity of the results we adopted, where available, the spectrophotometric distances given by Brand \& Blitz (1993). Some other references to the distance estimates are given in Table 1. The galacto-centric distances were calculated using the standard IAU value for $R_{0}$ of $8.5 \mathrm{kpc}$.

In Col. (10) we present the IRAS luminosities of our sources calculated as in Henning et al. (1990). 
Table 1. Source list. The letter "o" in the last column means an outflow detected as given by Wu et al. (1996) except S $76 \mathrm{E}$

\begin{tabular}{|c|c|c|c|c|c|c|c|c|c|c|}
\hline Name & $\begin{array}{l}\alpha(1950) \\
\left({ }^{\mathrm{h}}\right)\left({ }^{\mathrm{m}}\right)(\mathrm{s})\end{array}$ & $\begin{array}{l}\delta(1950) \\
\left(^{\circ}\right)\left(^{\prime}\right)\left(^{\prime \prime}\right)\end{array}$ & $\begin{array}{l}l \\
\left({ }^{\circ}\right)\end{array}$ & $\begin{array}{l}b \\
\left({ }^{\circ}\right)\end{array}$ & $\begin{array}{l}R_{\mathrm{G}} \\
(\mathrm{kpc})\end{array}$ & $\begin{array}{l}d \\
(\mathrm{kpc})\end{array}$ & $\begin{array}{l}z \\
(\mathrm{pc})\end{array}$ & IRAS PSC & $\begin{array}{l}L_{\mathrm{IR}} \\
\left(L_{\odot}\right)\end{array}$ & Remarks \\
\hline S 74 & 190614.9 & 053110 & 39.86 & -1.23 & 7.0 & $2.1^{a}$ & -45 & $19062+0531$ & $4.310^{3}$ & \\
\hline S $76 \mathrm{E}$ & 185346.2 & 074930 & 40.50 & +2.54 & 7.0 & $2.1^{b}$ & 93 & $18537+0749$ & $2.310^{4}$ & $\mathrm{o}^{h}, \mathrm{~W} 45$ \\
\hline S 86 & 194141.2 & 232146 & 59.64 & -0.18 & 7.7 & $1.9^{a}$ & -6 & $19416+2321$ & $2.410^{3}$ & \\
\hline S 87 & 194413.5 & 242800 & 60.88 & -0.13 & 7.6 & $2.3^{c}$ & -5 & $19442+2427$ & $4.010^{4}$ & $\mathrm{o}$ \\
\hline S $88 \mathrm{~B}$ & 194441.4 & 250517 & 61.48 & +0.10 & 7.7 & $2.0^{c}$ & 3 & $19446+2505$ & $7.710^{4}$ & $\mathrm{o}$ \\
\hline S 90 & 194705.2 & 264321 & 63.12 & +0.44 & 7.6 & $4.0^{a}$ & 31 & $19470+2643$ & $2.010^{4}$ & \\
\hline S 93 & 195256.0 & 270454 & 64.14 & -0.47 & 7.7 & $3.2^{a}$ & -26 & $19529+2704$ & $2.810^{4}$ & \\
\hline S 100 & 195950.0 & 332420 & 70.29 & +1.60 & 9.5 & $8.0^{a}$ & 223 & $19598+3324$ & $1.610^{6}$ & o, K3-50 \\
\hline S 145 & 222712.2 & 635821 & 108.19 & +5.52 & 8.8 & $0.9^{e}$ & 87 & $22272+6358 \mathrm{~A}$ & $6.210^{2}$ & o, L1206 \\
\hline S 146 & 224730.9 & 593903 & 108.20 & +0.58 & 10.9 & $4.7^{g}$ & 48 & $22475+5939$ & $4.410^{4}$ & \\
\hline S $161 \mathrm{~B}$ & 231401.9 & 612122 & 111.89 & +0.88 & 9.9 & $2.8^{a}$ & 43 & $23140+6121$ & $5.210^{3}$ & \\
\hline S 199 & 025735.6 & 601722 & 138.30 & +1.56 & 10.2 & $2.1^{a}$ & 57 & $02575+6017$ & $9.310^{3}$ & o, IC $1848 \mathrm{~A}$ \\
\hline S 201 & 025920.6 & 601608 & 138.50 & +1.64 & 10.2 & $2.1^{i}$ & 60 & $02593+6016$ & $1.110^{4}$ & \\
\hline S 231 & 053548.8 & 354341 & 173.47 & +2.55 & 10.8 & $2.3^{a}$ & 102 & $05358+3543$ & $8.510^{3}$ & $\mathrm{o}$ \\
\hline S 255 & 060957.9 & 180012 & 192.60 & -0.05 & 11.0 & $2.5^{a}$ & -2 & $06099+1800$ & $4.710^{4}$ & o, S254/S258 \\
\hline RNO $1 \mathrm{~B}$ & 003353.3 & 631232 & 121.30 & +0.66 & 9.4 & $0.85^{f}$ & 18 & $00338+6312$ & $5.410^{2}$ & o, L1287 \\
\hline BFS 48 & 054804.8 & 254529 & 183.35 & -0.58 & 10.6 & $2.1^{d}$ & -21 & $05480+2545$ & $3.410^{3}$ & \\
\hline
\end{tabular}

${ }^{a}$ Brand \& Blitz (1993); ${ }^{b}$ Plume et al. (1992); ${ }^{c}$ Blitz et al. (1982); ${ }^{d}$ Henning et al. (1992); ${ }^{e}$ Avedisova \& Palous (1989); ${ }^{f}$ Yang et al. (1991); ${ }^{g}$ Fich \& Blitz (1984); ${ }^{h}$ Mattila et al. (1996); ${ }^{i}$ assumed.

\subsection{Data reduction and analysis}

We have reduced the data and produced maps using the GAG (Groupe d'Astrophysique de Grenoble) software package. The measured spectra were fitted by the function $\sum A_{i}(\nu)\left\{1-\exp \left[-\sum \tau_{j}(\nu)\right]\right\}\left(\tau_{j}\right.$ is the optical depth in the $j$-th component) describing one or more ammonia hfs patterns assuming gaussian velocity distributions and equal widths of the hfs components in each pattern. The derived parameters were the line intensities, LSR velocities and FWHM for the opacity distribution. For the spectra of sufficiently good quality it was possible to derive the optical depth as a sum of the peak opacities of the main group of hyperfine components from this fit.

The next steps in the data analysis were the derivation of the rotation temperature $T_{12}$, kinetic temperature $T_{\mathrm{k}}$ and total ammonia column density $N\left(\mathrm{NH}_{3}\right)$. These estimates have been made according to the procedure described by Harju et al. (1993).

From the ammonia column densities we derived hydrogen column densities and masses of the cores assuming the $\mathrm{NH}_{3}$ relative abundance of $\chi=310^{-8}$ (see the discussion by Harju et al.).

We estimated the source areas $(A)$ by integrating the column densities over the maps presented in Sect. 3 and dividing these values by the peak column densities. The angular sizes were found then from $\theta=\sqrt{(4 / \pi) A}$. It is worth mentioning that for a gaussian brightness distribution this estimate gives the size at the $\mathrm{e}^{-1}$ level which is by a factor of $1 / \sqrt{\ln 2}$ larger than the size at the half-power level. Then the linear dimensions $(L)$ were determined us- ing the distances from Table 1. Some sources are elongated so that these sizes represent geometric mean values between their axes. We attempted to take into account the beam size in an approximate way (as in Zinchenko 1995). We use "deconvolved" sizes defined as $\theta_{\text {deconv }}=\sqrt{\theta^{2}-\theta_{\mathrm{A}}^{2}}$ where $\theta_{\mathrm{A}}$ is the antenna beam width at the corresponding level. Of course, this procedure accounts for the beam size only approximately because many cores are elongated or have a rather complex brightness distribution. The density estimates have been corrected accordingly. Actually, these corrections do not exceed a few percent.

The mean densities were obtained as the peak column densities divided by these sizes. The masses were estimated from the integrals of the column densities. A factor of 1.36 (the ratio of total gas mass to hydrogen mass, e.g. Hildebrand 1983) was applied to derive the total mass of the cloud. The average line widths are the widths of the average source spectra and include therefore velocity gradients in the source. The virial masses have been calculated as in Paper II.

\section{Results}

\subsection{Observational data}

Most of the detected sources were mapped in the ammonia lines. In Fig. 1 we present the grey-scale maps of the $\mathrm{NH}_{3}$ $(1,1)$ emission. The positions of the $\mathrm{H}_{2} \mathrm{O}$ masers (taken mainly from Palagi et al. 1993), IRAS point sources as well as near-IR sources from the CIO catalogue (Gezari et al. 1993) are indicated. We plot the following maps for 

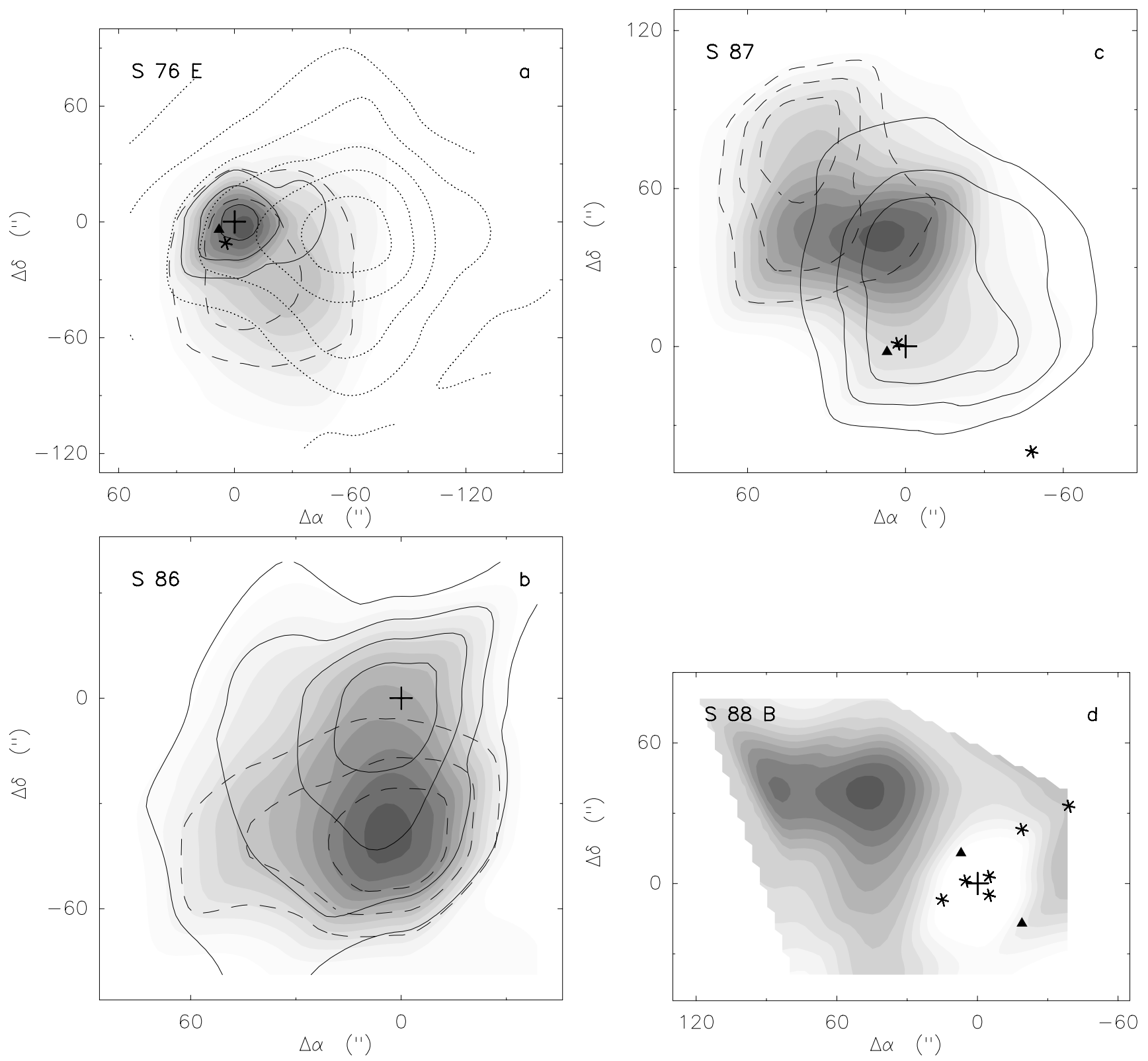

Fig. 1. The integrated $\mathrm{NH}_{3}(1,1)$ line intensity maps (grey-scale). The levels start from $15 \%$ of the peak intensities in steps of 7.5\%. The velocity ranges are indicated in Table 2. Solid and dashed contours correspond to the blue- and red-shifted velocity intervals, respectively (see text). The levels for these contours are the following: from $1 \mathrm{~K} \mathrm{~km} \mathrm{~s}^{-1}$ with $1 \mathrm{~K} \mathrm{~km} \mathrm{~s}^{-1}$ increment a), from $0.3 \mathrm{~K} \mathrm{~km} \mathrm{~s}^{-1}$ with $0.3 \mathrm{~K} \mathrm{~km} \mathrm{~s}^{-1}$ increment $\mathbf{b}$ ), from $0.5 \mathrm{~K} \mathrm{~km} \mathrm{~s}^{-1}$ with $0.5 \mathrm{~K} \mathrm{~km} \mathrm{~s}^{-1}$ increment $\mathbf{c}, \mathbf{g}, \mathbf{h}, \mathbf{j}$ ), from $0.2 \mathrm{~K} \mathrm{~km} \mathrm{~s}$ with $0.2 \mathrm{~K} \mathrm{~km} \mathrm{~s}^{-1}$ increment f), from $0.7 \mathrm{~K} \mathrm{~km} \mathrm{~s}^{-1}$ with $0.7 \mathrm{~K} \mathrm{~km} \mathrm{~s}^{-1}$ increment i) The crosses show the positions of the strong IRAS point sources listed in Table 1. The triangles indicate the positions of the $\mathrm{H}_{2} \mathrm{O}$ masers. The asterisks mark the positions of near-IR sources from the CIO catalogue. The dotted contours correspond to the CS $J=2-1$ maps from Paper II

most detected sources: maps of $\mathrm{NH}_{3}(1,1)$ line intensity integrated over the main group of the hfs components and $(1,1)$ maps for the blue- and red-side velocity intervals which represent $1 / 3$ of the total velocity range each. Thus, these maps give an impression of the source kinematics. To determine the total velocity range a composite spectrum was constructed for each source by overlaying all the measured $(1,1)$ spectra in one plot. Then, by visual in- spection of this composite spectrum the velocity range was determined as the interval where a noticeable emission in the main group was present above the $3 \sigma$ level (corresponding to $\sim 0.3 \mathrm{~K}$ ). For several sources CS $J=2-1$ maps from Paper II are overlayed on the ammonia maps. In Table 2 we present peak values of the $\mathrm{NH}_{3}(1,1)$ integrated line intensites and locations of the peaks as given by the GAG routines. In Cols. (5) and (6) of Table 2 the 

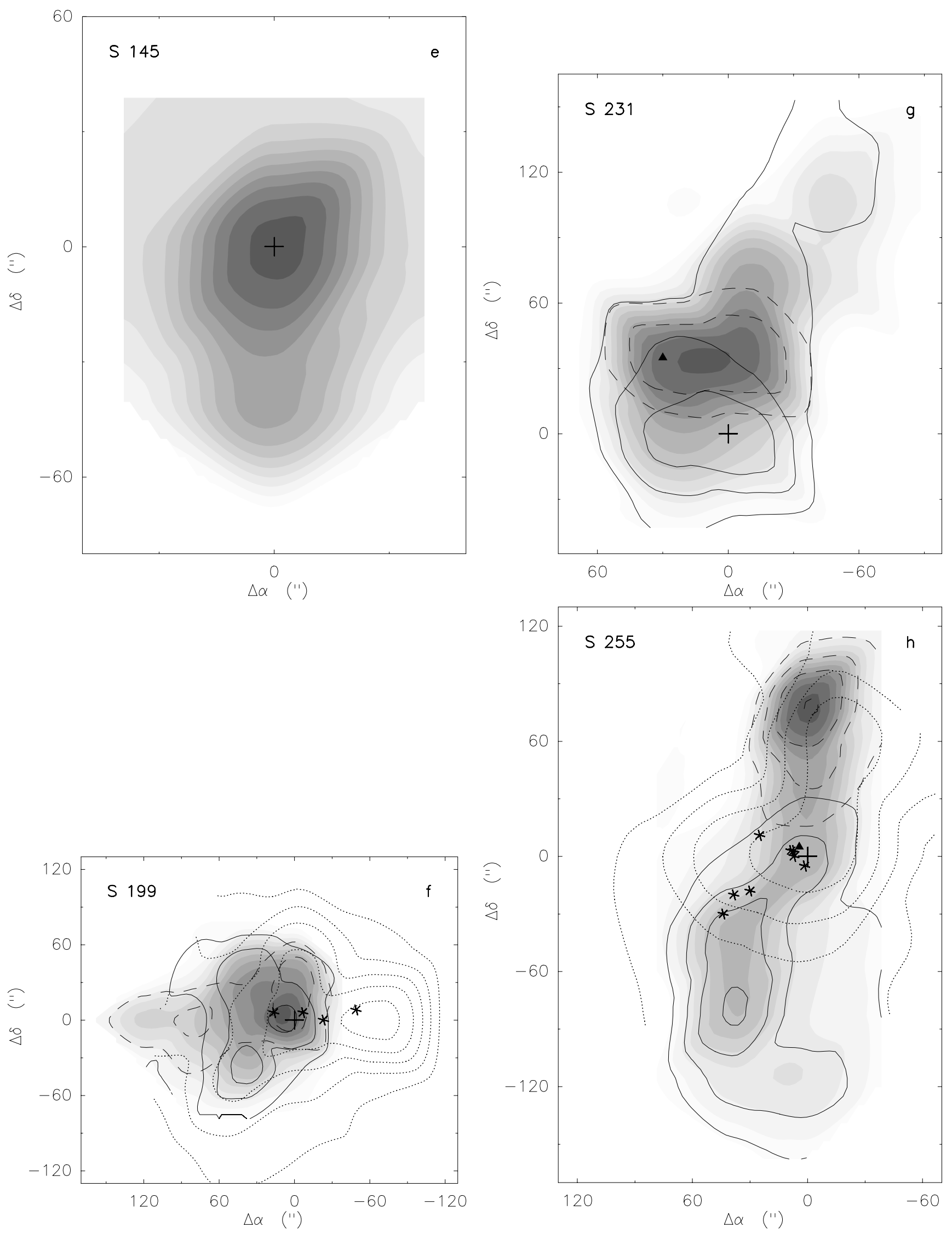

Fig. 1. continued 

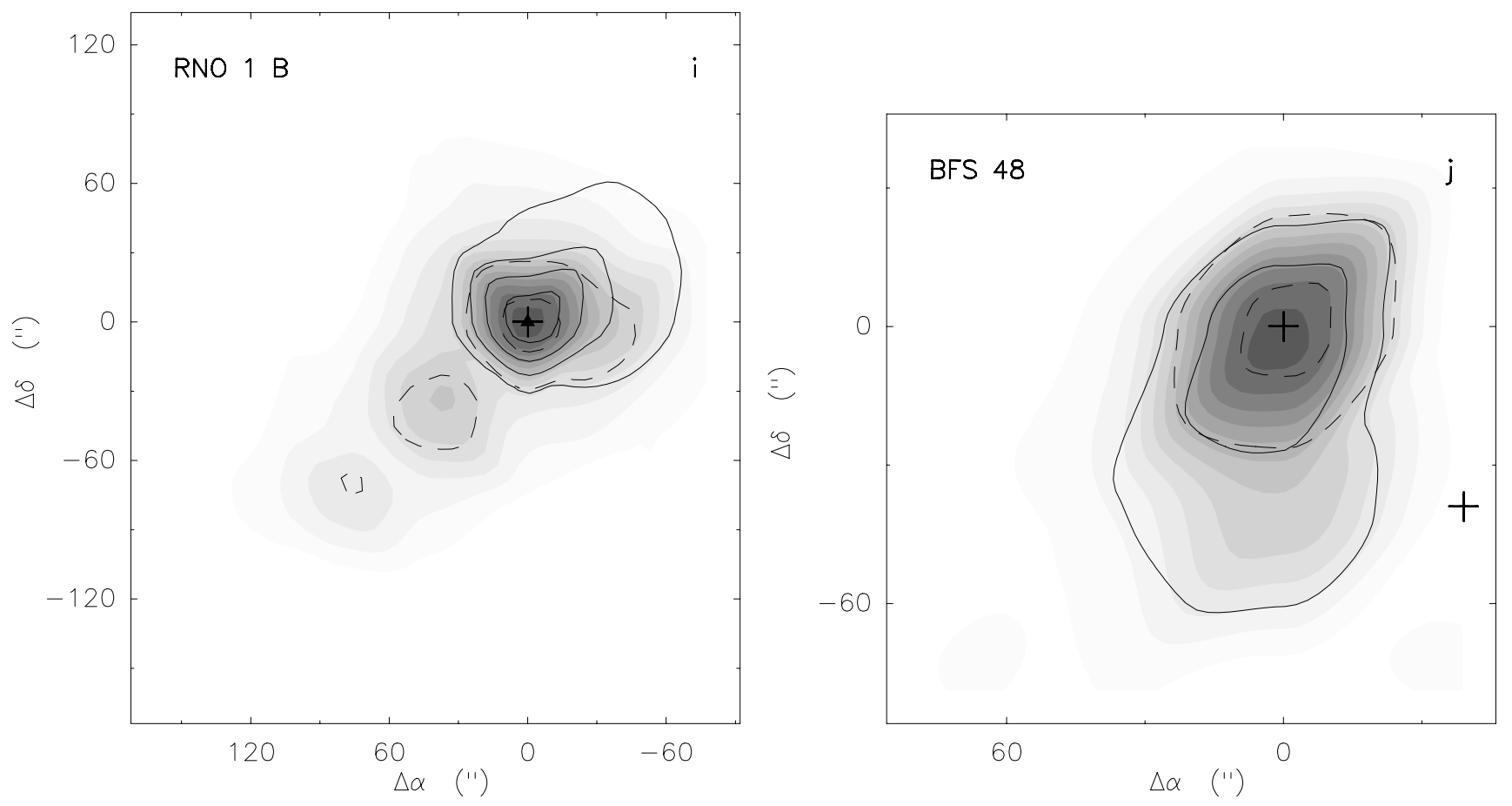

Fig. 1. continued

lower and upper boundaries of the velocity ranges used for integrating the line intensities $\left(V_{\min }\right.$ and $\left.V_{\max }\right)$ are indicated.

The $\mathrm{NH}_{3}(1,1)$ and $(2,2)$ line parameters at the grid positions closest to the peaks of the ammonia emission are presented in Table 3. The typical rms noise was

$\sim 0.1-0.15 \mathrm{~K}$ on the $T_{\mathrm{MB}}$ scale.

The non-detected sources are listed in Table 4 .

In addition, in Tables 5-14 we give some results describing the structure of the sources: velocities and line widths for the $(1,1)$ lines at the observed positions (obtained from the fitting procedure) as well as estimates of the $\mathrm{NH}_{3}$ column densities and kinetic temperatures. The latter are presented only for those positions where reliable estimates of the optical depth in the $(1,1)$ line could be obtained.

\subsection{Comments on individual objects}

\subsubsection{S $76 \mathrm{E}$}

This source shows a very unusual HCN emission spectrum in which the main component $(F=2-1)$ is practically absent (Zinchenko et al. 1993). Zinchenko et al. suggested that this effect can be caused by a foreground absorbing cloud with a velocity blue-shifted by $\sim 5 \mathrm{~km} \mathrm{~s}^{-1}$ relative to the $\mathrm{HCN}$ emitting core. In our $\mathrm{NH}_{3}(1,1)$ spectra (Fig. 2) a weak additional component can be seen.

In Fig. 3 we plot the average $\mathrm{NH}_{3}(1,1)$ spectrum in this source and the residual $\mathrm{NH}_{3}(1,1)$ spectrum obtained
Table 2. Peak values of $(1,1)$ integrated line intensities, locations of the peaks and boundaries of the velocity ranges used for integrating the line intensities

\begin{tabular}{lrrrrr}
\hline Source & $\begin{array}{r}\Delta \alpha_{\mathrm{p}} \\
\left({ }^{\prime \prime}\right)\end{array}$ & $\begin{array}{r}\Delta \delta_{\mathrm{p}} \\
\left(^{\prime \prime}\right)\end{array}$ & $\begin{array}{r}\int T_{\mathrm{MB}} \mathrm{d} v \\
\left(\mathrm{~K} \mathrm{~km} \mathrm{~s}^{-1}\right)\end{array}$ & $\begin{array}{r}V_{\min } \\
\left(\mathrm{km} \mathrm{s}^{-1}\right)\end{array}$ & $\begin{array}{r}V_{\max } \\
\left(\mathrm{km} \mathrm{s}^{-1}\right)\end{array}$ \\
\hline S 76 E & 0 & 0 & 12.9 & 29.4 & 35.1 \\
S 86 & 0 & -40 & 5.3 & 26.0 & 30.0 \\
S 87 & 10 & 40 & 7.6 & 20.0 & 27.0 \\
S 88 B & 50 & 40 & 1.2 & 20.0 & 24.0 \\
S 145 & 0 & 0 & 1.6 & -11.3 & -7.5 \\
S 199 & 10 & 10 & 2.2 & -39.5 & -36.5 \\
S 231 & 10 & 30 & 7.0 & -19.5 & -13.5 \\
S 255 & 0 & 80 & 4.7 & 4.8 & 10.8 \\
RNO 1B & 0 & 0 & 9.6 & -19.6 & -15.1 \\
BFS 48 & 0 & 0 & 5.9 & -11.5 & -7.0 \\
\hline
\end{tabular}

by subtracting the single gaussian $\mathrm{NH}_{3}$ pattern fit from the average spectrum. It clearly shows a weak $\mathrm{NH}_{3}$ line with a central velocity of $\sim 28 \mathrm{~km} \mathrm{~s}^{-1}$ and a width of $\sim 3 \mathrm{~km} \mathrm{~s}^{-1}$. This detection strongly supports the hypothesis of the foreground cloud mentioned above. There is no sign of this feature in the $\mathrm{NH}_{3}(2,2)$ spectra, which implies an upper limit on the kinetic temperature of $\sim 15 \mathrm{~K}$. The inspection of our data shows that the intensity of this component grows from our central position towards the south-west. However, the fact that this component is especially pronounced in the average spectrum indicates 
Table 3. $\mathrm{NH}_{3}(1,1)$ and $(2,2)$ line parameters at the grid positions closest to the emission peaks. The numbers in the brackets are the statistical uncertainties in the last digits (standard deviations). In the last column kinetic temperatures derived from these data are indicated

\begin{tabular}{|c|c|c|c|c|c|c|c|c|c|c|}
\hline \multirow[b]{2}{*}{ Source } & \multirow[b]{2}{*}{$\begin{array}{l}\Delta \alpha \\
\left({ }^{\prime \prime}\right)\end{array}$} & \multirow[b]{2}{*}{$\begin{array}{l}\Delta \delta \\
\left({ }^{\prime \prime}\right)\end{array}$} & \multicolumn{4}{|l|}{$(1,1)$} & \multicolumn{3}{|l|}{$(2,2)$} & \multirow[b]{2}{*}{$\begin{array}{l}T_{\mathrm{k}} \\
(\mathrm{K})\end{array}$} \\
\hline & & & $\begin{array}{l}T_{\mathrm{MB}} \\
(\mathrm{K})\end{array}$ & $\begin{array}{l}V_{\mathrm{LSR}} \\
\left(\mathrm{km} \mathrm{s}^{-1}\right)\end{array}$ & $\begin{array}{l}\Delta V \\
\left(\mathrm{~km} \mathrm{~s}^{-1}\right)\end{array}$ & $\tau$ & $\begin{array}{l}T_{\mathrm{MB}} \\
(\mathrm{K})\end{array}$ & $\begin{array}{l}V_{\mathrm{LSR}} \\
\left(\mathrm{km} \mathrm{s}^{-1}\right)\end{array}$ & $\begin{array}{l}\Delta V \\
\left(\mathrm{~km} \mathrm{~s}^{-1}\right)\end{array}$ & \\
\hline S $76 \mathrm{E}$ & 0 & 0 & $3.96(07)$ & $32.04(01)$ & $2.70(0.03)$ & $0.83(0.05)$ & $2.63(22)$ & $32.09(02)$ & $2.91(0.09)$ & $27.2(1.3)$ \\
\hline S 86 & 0 & -40 & $2.65(21)$ & $28.01(02)$ & $1.48(0.07)$ & $1.46(0.27)$ & $1.87(44)$ & $28.06(05)$ & $1.62(0.17)$ & $26.0(3.5)$ \\
\hline \multirow[t]{2}{*}{ S 87} & 0 & 40 & $2.93(16)$ & $23.65(02)$ & $1.48(0.05)$ & $1.45(0.19)$ & $1.96(07)$ & $23.65(30)$ & $1.61(1.03)$ & $25.0(3.0)$ \\
\hline & 0 & 40 & $1.31(16)$ & $21.14(02)$ & $0.81(0.11)$ & $2.15(0.73)$ & $0.72(07)$ & $21.25(30)$ & $0.89(1.03)$ & $18.8(3.5)$ \\
\hline S 88 B & 40 & 40 & $0.45(14)$ & $21.58(16)$ & $2.50(0.39)$ & & $0.38(06)$ & $21.43(21)$ & $2.28(0.45)$ & $38(10)$ \\
\hline S 145 & 0 & 0 & $1.08(09)$ & $-9.77(04)$ & $1.22(0.13)$ & & $0.45(08)$ & $-9.96(13)$ & $1.71(0.39)$ & $22(04)$ \\
\hline S 199 & 0 & 0 & $1.23(12)$ & $-38.17(03)$ & $1.48(0.09)$ & $0.66(0.28)$ & $0.81(07)$ & $-38.19(05)$ & $1.55(0.15)$ & $27.9(2.1)$ \\
\hline S 201 & 40 & 0 & $0.76(13)$ & $-37.74(09)$ & $1.16(0.23)$ & & $0.41(11)$ & $-37.91(25)$ & $2.32(0.73)$ & $54(27)$ \\
\hline S 231 & 30 & 35 & $2.43(10)$ & $-16.37(02)$ & $2.16(0.06)$ & $0.97(0.13)$ & $1.61(22)$ & $-16.24(03)$ & $1.90(0.11)$ & $26.5(2.1)$ \\
\hline \multirow[t]{2}{*}{ S 255} & 0 & 80 & $1.64(11)$ & $8.65(03)$ & $2.41(0.09)$ & $0.85(0.19)$ & $0.90(04)$ & $8.69(05)$ & $2.61(0.15)$ & $23.0(1.0)$ \\
\hline & 40 & -40 & $1.34(14)$ & $6.55(04)$ & $1.83(0.11)$ & $0.54(0.28)$ & $0.75(06)$ & $6.60(08)$ & $1.91(0.21)$ & $24.7(2.5)$ \\
\hline RNO $1 \mathrm{~B}$ & 0 & 0 & $3.85(16)$ & $-17.65(02)$ & $2.09(0.05)$ & $0.98(0.13)$ & $1.93(46)$ & $-17.74(04)$ & $2.21(0.18)$ & $21.1(2.3)$ \\
\hline BFS 48 & 0 & 0 & $2.53(17)$ & $-9.36(03)$ & $1.85(0.07)$ & $1.35(0.23)$ & $1.16(32)$ & $-9.36(08)$ & $1.99(0.21)$ & $18.7(2.2)$ \\
\hline
\end{tabular}

that its spatial extension is not much less than the size of the mapped region.

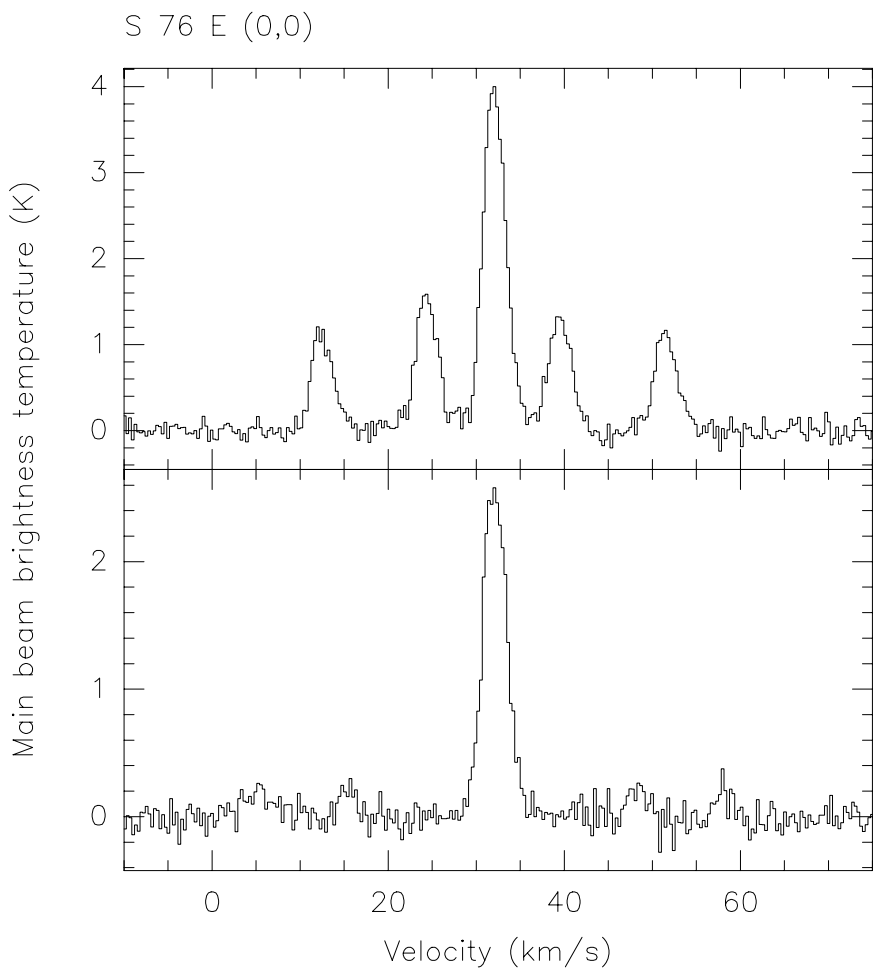

Fig. 2. The $\mathrm{NH}_{3}(1,1)$ and $(2,2)$ lines measured at the central position in $\mathrm{S} 76 \mathrm{E}$

It is worth noting that $\mathrm{S} 76 \mathrm{E}$ is the strongest source of ammonia emission in our sample. The maps in different
Table 4. List of non-detected sources. Rms noise per channel is indicated

\begin{tabular}{ll}
\hline Source & $\begin{array}{l}\sigma \\
(\mathrm{K})\end{array}$ \\
\hline S 74 & 0.16 \\
S 90 & 0.16 \\
S 93 & 0.17 \\
S 100 & 0.23 \\
S 146 & 0.20 \\
S 161 B & 0.20 \\
\hline
\end{tabular}

velocity intervals reveal a slight velocity gradient approximately in the north-south direction.

\subsubsection{S 87}

Our data clearly show the presence of two kinematically distinct components which overlap partly spatially (Figs. 1, 4). Similar structure has been seen in some other lines (e.g. Barsony 1989) including $\mathrm{NH}_{3}$ (Stutzki et al. 1984). However, to our knowledge the source has not been mapped in the ammonia lines yet. A submillimeter continuum map at $850 \mu \mathrm{m}$ (Jenness et al. 1995) shows a morphology which is very similar to our ammonia map.

\subsubsection{RNO $1 \mathrm{~B}$}

This cloud was subject to detailed investigations in various molecular lines including the ammonia lines by Estalella et al. (1993) and Yang et al. (1991, 1995). Our ammonia data are very similar to the results obtained by Estalella 


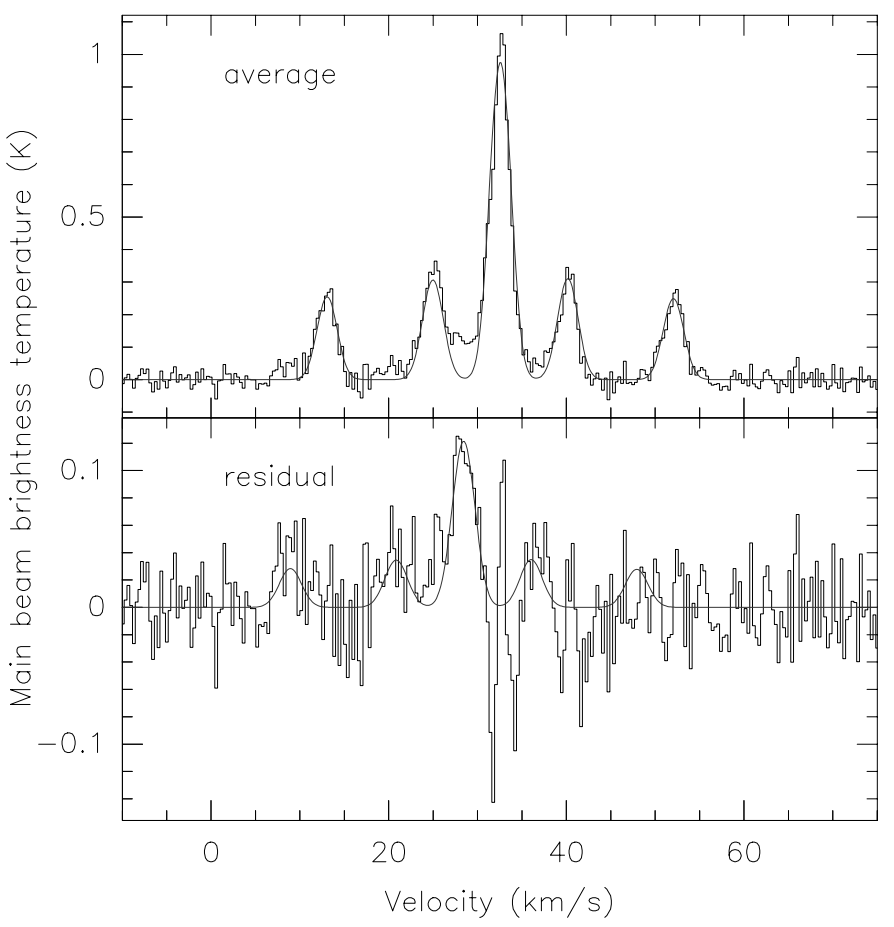

Fig. 3. The average $\mathrm{NH}_{3}(1,1)$ spectrum in $\mathrm{S} 76 \mathrm{E}$ and the residual spectrum obtained by subtraction of the single $\mathrm{NH}_{3}$ gaussian pattern from the average spectrum

\section{S $87\left(0,40^{\prime \prime}\right)$}

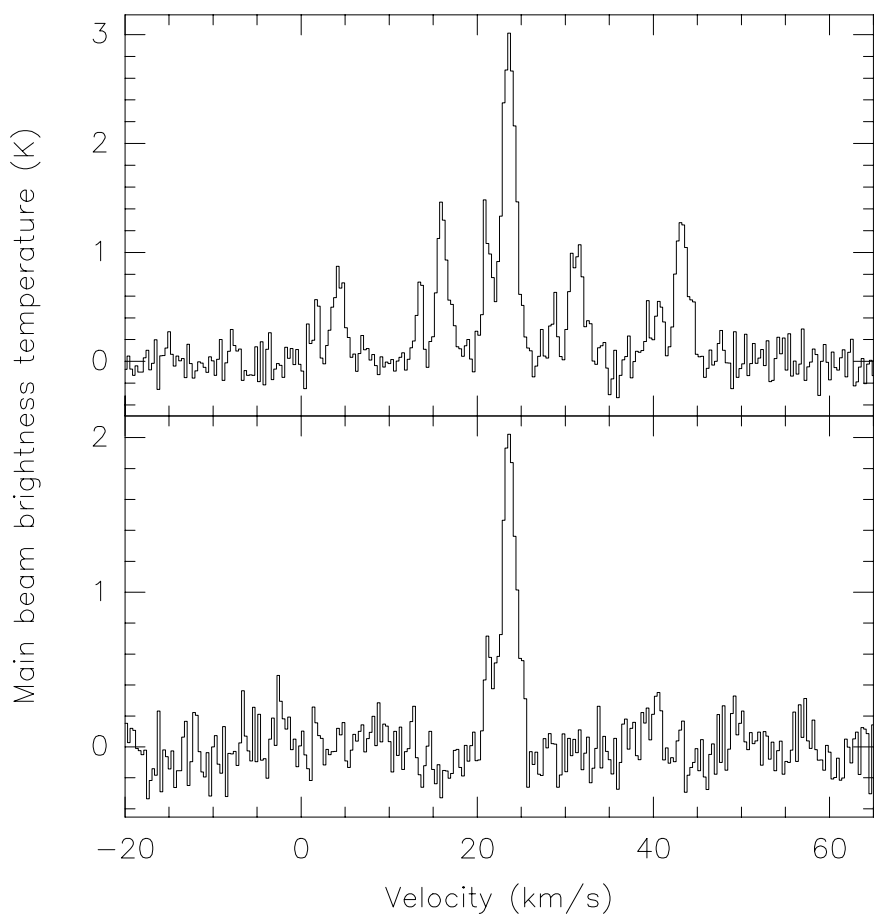

Fig. 4. The $\mathrm{NH}_{3}(1,1)$ and $(2,2)$ lines measured at the position $\left(0,40^{\prime \prime}\right)$ in $\mathrm{S} 87$ et al. and we present them here just for the sake of the homogeneity for statistical analysis.

\subsection{Physical properties of the sources}

In Table 3 we present the values of the kinetic temperatures at the grid positions closest to the peaks of the ammonia emission. We estimate the uncertainties of these values in $\sim 2 \mathrm{~K}$ in most cases. The exceptions are $\mathrm{S} 88 \mathrm{~B}$, S 145 and S 201 where the lines are too weak. For them we give kinetic temperatures derived in the optically thin approximation from the ratio of the $(2,2)$ and $(1,1)$ line areas (Harju et al. 1993).

The sizes, masses, mean densities, average line widths and virial masses determined in the way described in Sect. 2.3 are presented in Table 15 .

Table 15. Sizes, masses, mean densities, average line widths and virial masses of the clouds

\begin{tabular}{llllll}
\hline Source & $\begin{array}{l}L \\
(\mathrm{pc})\end{array}$ & $\begin{array}{l}\log \bar{n} \\
\left(\mathrm{~cm}^{-3}\right)\end{array}$ & $\begin{array}{l}M \\
\left(M_{\odot}\right)\end{array}$ & $\begin{array}{l}\overline{\Delta V} \\
\left(\mathrm{~km} \mathrm{~s}^{-1}\right)\end{array}$ & $\begin{array}{l}M_{\text {vir }} \\
\left(M_{\odot}\right)\end{array}$ \\
\hline S 76 E & 0.91 & 4.26 & 700 & 2.63 & 660 \\
S 86 & 0.64 & 4.12 & 180 & 1.52 & 160 \\
S 87 & 0.94 & 4.08 & 530 & 3.30 & 1080 \\
S 88 B & 1.10 & 3.02 & 74 & 2.42 & 680 \\
S 145 & 0.40 & 3.63 & 14 & 1.22 & 62 \\
S 199 & 1.28 & 3.31 & 230 & 1.36 & 250 \\
S 231 & 1.24 & 3.84 & 690 & 1.96 & 500 \\
S 255 & 1.43 & 3.60 & 600 & 3.00 & 1350 \\
RNO 1 B & 0.48 & 4.42 & 150 & 1.65 & 140 \\
BFS 48 & 0.75 & 4.10 & 270 & 1.85 & 270 \\
\hline
\end{tabular}

The comparison with the CS $J=2-1$ results from Paper II for S 76 E, S 199 and S 255 shows that the ammonia lines are $\sim 30 \%$ narrower than the CS lines. The source sizes are $\sim 2$ times smaller in ammonia. The core masses are correspondingly smaller. But the mean densities are similar. This is similar to the results of such comparison for other clouds in Paper II.

\section{Discussion}

\subsection{Ammonia as a tracer of dense gas}

The ammonia emission was detected in about half of the sources in the range of right ascension of $12^{\mathrm{h}}-24^{\mathrm{h}}$. This is comparable with the detection rate obtained in Paper I for the outer Galaxy (37\%) but the number of sources in the present study is certainly insufficient for statistical conclusions.

Comparing the detected and non-detected sources we should mention two apparent differences between these groups: (1) the non-detected sources are significantly more 
distant on the average than the detected ones (the mean distances are $4.1 \pm 0.9 \mathrm{kpc}$ and $1.9 \pm 0.2 \mathrm{kpc}$, respectively) and (2) the detection rate for the sources with outflows is much higher than for the sources without reported outflows (8 from 9 and 3 from 8, respectively). However, the second item can easily be a consequence of the first one because it should be more difficult to detect outflows in more distant objects. Therefore, we cannot conclude whether the detection rate is related to the outflow activity. Perhaps the differences can be explained by the beam dilution effects. Further we shall concentrate mostly on the detected objects.

The masses derived from the column densities are remarkably close to virial masses in most cases. It shows in particular that our assumption of the ammonia abundance, $\chi\left(\mathrm{NH}_{3}\right)=310^{-8}$, is in general correct (at least for the detected clouds). The striking exception is $\mathrm{S} 88 \mathrm{~B}$. The mass derived for this source is an order of magnitude lower than the virial mass. This may indicate that the ammonia abundance here is correspondingly lower than in most of the other detected cores. The map of the ammonia emission in S 88 B (Fig. 1) shows a "hole" at the IR source position where we detected no ammonia at all. This resembles the distribution of the $\mathrm{CO}$ emission which has a horseshoe-like structure (White \& Fridlund 1992) with a significantly reduced intensity of the $\mathrm{CO}$ line in approximately the same region. However, it is worth noting that the CS emission towards the IR source observed with a similar beam size (though not mapped) is rather strong (Paper II).

For explanation of the apparent ammonia underabundance it might be important that the IRAS point source associated with $\mathrm{S} 88 \mathrm{~B}$ is very luminous $\left(\sim 10^{5} L_{\odot}\right)$, second in our sample by the luminosity. The most luminous source in the sample is S 100 (K 3-50) and we detected no ammonia emission in this object (earlier Churchwell et al. 1990) observed a weak ammonia emission here; the intensity was lower than our detection limit). However, $\mathrm{K}$ 3-50 is a strong source of molecular emission in the lines of other high density tracers, e.g. HCN $J=1-0$ (Burov et al. 1988) and CS $J=7-6$ (Plume et al. 1992).

For S 87 and S 255 the virial masses also exceed (by a factor of 2) the masses derived from the ammonia column densities. Both these sources have pronounced bipolar structure and can be really unbound. It is worth noting also that the estimates of the virial masses in the case of their highly elongated geometry should be made in a more refined way.

The difference between the ammonia distribution on the one hand and CS and dust distribution on the other hand in S 255 (which is also very luminous) can also be attributed to the ammonia underabundance in the neighbourhood of the central source.

Thus, we suspect that ammonia is underabundant in the vicinity of the most powerful IR sources. Of course, this is not a decisive conclusion because the number of the investigated objects is too limited and the beam is pretty large. Recently Davis \& Dent (1993) suggested that ammonia is underabundant towards young stars being the central sources of molecular outflows but did not relate this effect with the IR luminosity. However, many of our sources demonstrate outflow activity but mainly the most luminous ones have signs of ammonia underabundance.

What can be the reason for the ammonia underabundance? Davis \& Dent (1993) suggested that there is a mechanism which restricts ammonia formation in such places. In our opinion there are basically two ways to explain the possible ammonia underabundance:

(1) The time-dependent chemical models show that the ammonia abundance is relatively low at early times (e.g. Bergin et al. 1995; Taylor et al. 1996). Thus, the ammonia underabundance can be related to the evolutionary status of the clouds. However, there is no direct relation to the luminosity of the central source in this model.

(2) An enhanced radiation field in the vicinity of young stellar objects increases the thickness of the $\mathrm{C}^{+}$layer and $\mathrm{NH}_{3}$ is destroyed primarily by a reaction with $\mathrm{C}^{+}$(e.g. Turner 1995). At the same time the species like CO, CN, $\mathrm{C}_{2} \mathrm{H}$, etc. which are formed via $\mathrm{C}^{+}$are still very abundant in these regions in spite of the high photodestruction rates (Bergin et al. 1995; Jansen et al. 1995). Probably this difference in their chemistry under the conditions of enhanced radiation field is the most plausible explanation for the relative ammonia underabundance.

\subsection{Spatial structure and kinematics}

About half of the mapped sources show elongated structures with a more or less significant velocity gradient along the major axis. The most prominent example is S 255 . S 87 has a two-component structure with 2 distinct velocity components which overlap partly spatially. However, as it has been argued above ammonia probably does not reflect the dense gas distribution correctly especially in these cases. Anyway, there is a question what is the reason for this appearance. The most probable explanations are a rotating disk or an outflow. Barsony (1989) argued in favour of the latter model for S 87 . We shall discuss the data on these sources in more detail in a separate paper.

Another important item in respect of the core kinematics is the variations of the velocity dispersion in the cores. The CS data for southern cores with an apparently spherical structure indicate that the velocity dispersion decreases outwards with radius (Zinchenko 1995). The present ammonia data are not so definite. In S 199, S 231, RNO 1B and BFS 48 the line widths increase significantly near the ammonia peaks. In S 87 the widths of the separate components also increase near their corresponding emission peaks. In the other cases there are either no regular variations (S 86) or gradients of the line widths along certain directions can be seen (S 76E, S 88B, S 255). But 
most of the cores in the present sample are far from the spherical symmetry.

\subsection{Temperature}

Considering the kinetic temperature distribution in the cores we see that in most cases it is centrally peaked reaching $20-28 \mathrm{~K}$ and drops to $15-20 \mathrm{~K}$ at the edges of the ammonia emitting regions. These values refer to the $40^{\prime \prime}$ beam of the Effelsberg antenna and do not exclude a presence of hotter compact clumps. Probably, in the outer layers of the clouds which are not traced by ammonia, the temperature rises again as indicated by the fact that the CO temperatures in many cases are higher than the kinetic temperatures derived from ammonia data.

\subsection{Hyperfine intensity anomalies}

Finally, we have looked for hyperfine intensity anomalies in the $(1,1)$ transition in our sources. In addition to $\mathrm{S} 87$ where such anomalies have been seen by Stutzki et al. (1984) we found similar deviations in S 199 (Fig. 5). These anomalies are a rather common phenomenon in warm clouds (Stutzki \& Winnewisser 1985) and have been interpreted in the framework of clumpy models with the individual clumps having small line widths of $\sim 0.3-0.6 \mathrm{~km} \mathrm{~s}^{-1}$ (see Stutzki \& Winnewisser 1985 and references therein).

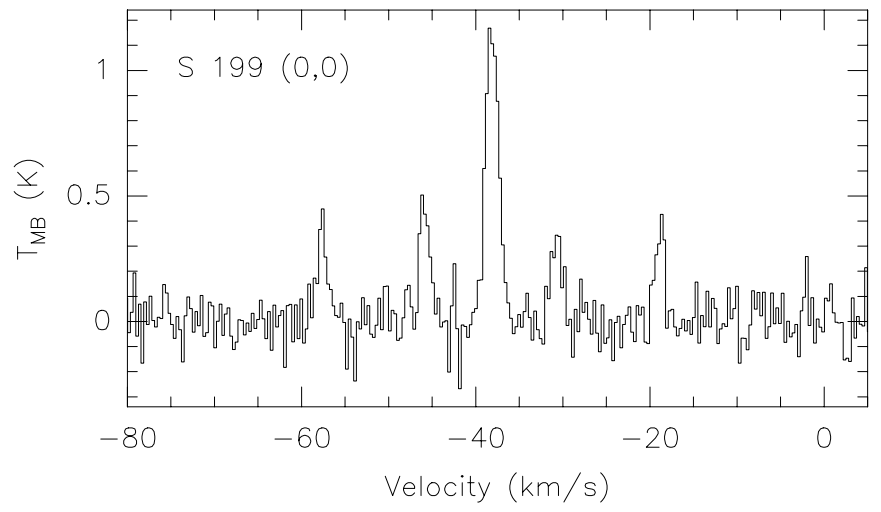

Fig. 5. The $\mathrm{NH}_{3}(1,1)$ line in $\mathrm{S} 199$ showing hyperfine intensity anomalies

\section{Summary and conclusions}

We have presented results of the observations of 17 molecular clouds associated with bright FIR sources in the $\mathrm{NH}_{3}$ $(1,1)$ and $(2,2)$ lines with the 100 -m radio telescope in Effelsberg. The lines were detected in 11 clouds and 10 of them have been mapped in these lines. The kinetic temperatures, sizes, masses and mean densities of the ammonia cores have been derived.
For most of the detected clouds the masses derived under the assumption of a relative ammonia abundance of $\chi\left(\mathrm{NH}_{3}\right)=310^{-8}$ are close to virial masses. However, in $\mathrm{S} 88 \mathrm{~B}$ the ratio $M / M_{\text {vir }}$ is only $\sim 0.1$ and in a few other cases this ratio is significantly lower than unity which may indicate ammonia underabundance. Almost all objects with the signs of underabundance are among the most luminous IR sources in our sample.

Most of the mapped cores are elongated with noticeable velocity gradients along the major axis. The highest elongation and velocity gradient are observed in S 255. S 87 has a two-component structure with 2 distinct velocity components which overlap partly spatially. In about a half of the mapped sources the $\mathrm{NH}_{3}$ line widths increase near the peaks of the ammonia emission.

The kinetic temperatures are centrally peaked. They reach $20-28 \mathrm{~K}$ in the center and drop to $15-20 \mathrm{~K}$ at the edges of the ammonia emitting regions.

In $\mathrm{S} 76 \mathrm{E}$ a weak extended emission in the $(1,1)$ line at the velocity blue-shifted by $\sim 5 \mathrm{~km} \mathrm{~s}^{-1}$ relative to the core emission was detected. Hyperfine intensity anomalies in the $(1,1)$ transition are noticeable in S 199 in addition to $\mathrm{S} 87$ which is a known case of these anomalies.

Acknowledgements. We thank J. Harju and C. Henkel for the helpful discussions, W.J. Altenhoff for providing calibration data for the 100-m antenna, K. Mattila for critical reading the manuscript and the anonymous referee for valuable suggestions which improved the presentation of the results. Special thanks are to C.M. Walmsley for many fruitful discussions and invaluable assistance at various stages of this work. I.Z. is very grateful to the MPG-AG "Staub in Sternentstehungsgebieten" for the hospitality. He was also supported in part by the INTAS grant 93-2168 and grant 94-02-04861-a from the Russian Foundation for Basic Research.

\section{References}

Avedisova V.S., Palouš J., 1989, Bull. Astron. Inst. Czechosl. 40,42

Barsony M., 1989, ApJ 345, 268

Bergin E.A., Langer W.D., Goldsmith P.F., 1995, ApJ 441, 222

Blitz L., Fich M., Stark A.A., 1982, ApJS 49, 183

Brand J., Blitz L., 1993, A\&A 275, 67

Burov A.B., Vdovin V.F., Zinchenko I.I., et al., 1988, PAZh 14, 492 (SvA Lett. 14, 209)

Carpenter J.M., Snell R.L., Schloerb F.P., 1990, ApJ 362, 147

Carpenter J.M., Snell R.L., Schloerb F.P., Skrutskie M.F., 1993, ApJ 407, 657

Churchwell E., Walmsley C.M., Cesaroni R., 1990, A\&AS 83, 119

Davis C.J., Dent W.R.F., 1993, MNRAS 261, 371

Estalella R., Mauersberger R., Torrelles J., et al., 1993, ApJ 419,698

Fich M., Blitz L., 1984, ApJ 279, 125

Fich M., Blitz L., Stark A.A., 1989, ApJ 342, 272

Gezari D.Y., Schmitz M., Pitts P.S., Mead J.M., 1993, Catalog of Infrared Observations, NASA Reference Publication 1294 
Harju J., Walmsley C.M., Wouterloot J.G.A., 1993, A\&AS 98, 51

Henning T., Pfau W., Altenhoff W.J., 1990, A\&A 227, 542

Henning T., Cesaroni R., Walmsley C.M., Pfau W., 1992, A\&AS 93, 525

Hildebrand H., 1983, QJRAS 24, 267

IRAS Catalogs, 1988, Infrared Astronomical Satellite (IRAS) Catalogs and Atlases, NASA, RP-1190

Jansen D.J., van Dishoeck E.F., Black J.H., Spaans M., Sosin C., 1995, A\&A 302, 223

Jenness T., Scott P.F., Padman R., 1995, MNRAS 276, 1024

Mattila K., et al., 1996 (in preparation)

Ott M., Witzel A., Quirrenbach A., et al., 1994, A\&A 284, 331

Palagi F., Cesaroni R., Comoretto G., Felli M., Natale V., 1993, A\&AS 101, 153

Pirogov L., Zinchenko I., Lapinov A., Myshenko V., Shulga V., 1995, A\&AS 109, 333

Plume R., Jaffe D.T., Evans II N.J., 1992, ApJS 78, 505

Schreyer K., Henning Th., Kömpe C., Harjunpää P., 1996, A\&A 306, 267 (Paper I)

Snell R.L., Huang Y.-L., Dickman R.L., Claussen M.J., 1988, ApJ 325, 853

Snell R.L., Dickman R.L., Huang Y.-L., 1990, ApJ 352, 139

Solomon P.M., Rivolo A.R., Barrett J., Yahil A., 1987, ApJS 63,821
Stutzki J., Winnewisser G., 1985, A\&A 144, 13

Stutzki J., Jackson J.M., Olberg M., Barrett A.H., Winnewisser G., 1984, A\&A 139, 258

Taylor S.D., Morata O., Williams D.A., 1996, A\&A 313, 269

Turner B.E., 1995, ApJ 444, 708

Walmsley C.M., Güsten R., 1994, Conditions in regions of high mass star formation. In: Wilson T.L., Johnston K.J. (eds.) The Structure and Content of Molecular Clouds, Lecture Notes in Physics 439. Springer-Verlag, Berlin

White G.J., Fridlund C.V.M., 1992, A\&A 266, 452

Wu Y., Huang M., He J., 1996, A\&AS 115, 283

Yang J., Ohashi N., Fukui Y., 1995, ApJ 455, 175

Yang L., Umemoto T., Iwata T., Fukui Y., 1991, ApJ 373, 137

Zhou S., Wu Y., Evans N.J. II, 1989, ApJ 346, 168

Zhou S., Evans N.J. II, Güsten R., Mundy L.G., Kutner M.L., 1991, ApJ 372, 518

Zinchenko I., 1995, A\&A 303, 554

Zinchenko I.I., Lapinov A.V., Pirogov L.E., 1989, AZh 66, 1142 (SvA 33, 590)

Zinchenko I.I., Krasil'nikov A.A., Kukina E.P., Lapinov A.V., Pirogov L.E., 1990, AZh 67, 908 (SvA 34, 458)

Zinchenko I., Forsström V., Mattila K., 1993, A\&A 275, L9

Zinchenko I., Forsström V., Lapinov A., Mattila K., 1994, A\&A 288, 601 (Paper II) 